\title{
Adaptive Oscillators with Human-in-the-Loop: Proof of Concept for Assistance and Rehabilitation
}

\author{
Renaud Ronsse, Nicola Vitiello, Tommaso Lenzi, Jesse van den Kieboom, \\ Maria Chiara Carrozza, and Auke Jan Ijspeert
}

\begin{abstract}
Most recent findings in robot-assisted therapy suggest that the therapy is more successful if the patient actively participates to the movement ("assistance-as-needed"). In the present contribution, we propose a novel approach for designing highly flexible protocols based on this concept. This approach uses adaptive oscillators: a mathematical primitive having the capacity to learn the high-level features of a quasi-sinusoidal signal (amplitude, frequency, offset). Using a simple inverse model, we demonstrate that this method permits to synchronize with the torque produced by the user, such that the effort associated with the movement production is shared between the user and the assistance device, without specifying any arbitrary reference trajectory. Simulation results also establish the method relevance for helping patients with movement disorders. Since our method is specifically designed for rhythmic movements, the final target is the assistance/rehabilitation of locomotory tasks. As an initial proof of concept, this paper focuses on a simpler movement, i.e. rhythmic oscillations of the forearm about the elbow.
\end{abstract}

\section{INTRODUCTION}

Dedicated robotics platforms are nowadays developed for the rehabilitation of patients suffering from movement disorders, both at the upper and lower extremities [1], [2], [3]. These robot-assisted therapies are mainly developed in order to be less labor intensive for the therapists, such that more frequent and/or longer training sessions can be proposed to the patient, eventually leading to faster and better recovery. Patient motivation can further be assessed by incorporating entertainment features within the rehabilitation protocol. Recent findings moreover suggest that therapy is more successful if the patient actively participates to the movement, giving rise to the concept of "assistance-asneeded" [4], [5].

For the lower-limb, traditional robotics platforms for rehabilitation stay far away from this concept, driving the user's limbs along a pre-specified reference trajectory using stiff position control [6], [7]. This approach has three main drawbacks: (i) if the user stays passive, he/she will be moved anyway, such that his/her own effort is not promoted; (ii) the reference trajectory is captured from the gait pattern of one or several other healthy individual(s), and might therefore

This work was supported by the EU within the EVRYON Collaborative Project STREP (FP7-ICT-2007-3-231451).

R. Ronsse, J. van den Kieboom, and A.J. Ijspeert are with the Biorobotics Laboratory, Institute of Bioengineering, École Polytechnique Fédérale de Lausanne, CH-1015 Lausanne, Switzerland. e-mail: \{renaud.ronsse, jesse.vandenkieboom, auke.ijspeert\}@epfl.ch

N. Vitiello, T. Lenzi and M.C. Carrozza are with the ARTS lab, Scuola Superiore Sant'Anna, I-56025 Pontedera (Pisa), Italy. e-mail: $\{$ n.vitiello, t.lenzi, carrozza\}esssup.it

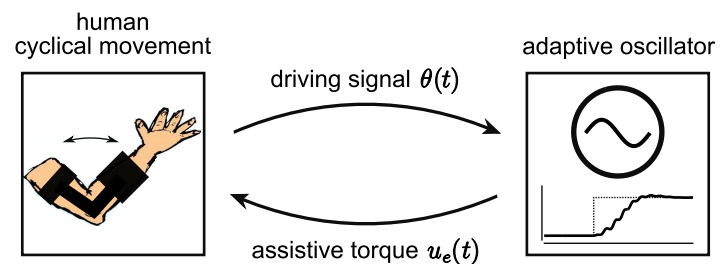

Fig. 1. Sketch of the synchronization between the human joint (elbow in this case) and an adaptive oscillator. The oscillator feeds back some torque $u_{e}(t)$ to the controlled joint.

not correspond to a suitable trajectory for the patient being trained; and (iii) making errors - which is not permitted by stiff position controllers - is often necessary for learning and the formation of an internal task representation within the motor repertoire [8]. More recently, rehabilitation protocols based on assistance-as-needed were proposed, both for the Lokomat [9] and other platforms [10] by adding some compliance with respect to the pre-specified trajectory.

In the present paper, we propose a novel assistance method for rhythmic movements that not only promotes the user's effort, in the sense that he/she will receive assistance only if making an effort on his/her own, but also allows the user to flexibly shape the intended trajectory. Indeed, there is actually no pre-specified trajectory by the experimenter, such that, in steady-state, the user should receive the same amount of assistance whatever the stabilized pattern. These two features are similar to what is achieved with EMG-based assistance devices [11], although these require complex sensing and very long calibration procedure to be optimally fitted to a single user [12]. In contrast, our method requires only to measure the assisted joint position.

The method is based on adaptive frequency oscillators ${ }^{1}$, that were developed by Righetti, Buchli, and Ijspeert [13], [14] for various applications [15]. Oscillator-based methods are very appealing in the context of rhythmic movement assistance, because these movements - like e.g. walking - are very likely controlled by spinal oscillators (Central Pattern Generators, CPGs) [16]. The "artificial" oscillator is thus assumed to synchronize with the spinal one, and in turn feeds back some torque to the controlled joint (Figure 1). Although our final target is to provide assistance during locomotory tasks, the present paper — as a proof

\footnotetext{
${ }^{1}$ For brevity, we will simply refer to adaptive oscillators in the rest of this paper. Furthermore, the dynamical system we are going to use as oscillator has the intrinsic capacity to adapt not only its frequency but also its amplitude and offset.
} 
of concept - will focus on simulations and experiments of rhythmic oscillations of the forearm about the elbow. We acknowledge that the intrinsic neural mechanisms to control the upper and lower limb are likely very distinct, but this simple configuration allows us to investigate the problem of assistance without having yet to deal with complex dynamics due to contacts with the ground and the coordination of multiple degrees of freedom.

The paper is organized as follows. In Section II, we provide a small recap of the adaptive oscillator used in this paper. In Section III, we provide both simulation and experimental results of movement assistance of the forearm for healthy participants. In Section IV, we discuss simulation results anticipating the method efficiency with a patient suffering from muscle weakness. The paper ends with a conclusion.

\section{ADAPTIVE OSCILLATOR: RECAP}

The adaptive oscillator used in this paper is directly adapted from [13], and was previously used for various different applications [15]. In the present case, the adaptive oscillator is used as state observer, in the sense that it acts like a filter to smoothen and anticipate the evolution of the corresponding joint state. Unlike conventional filters, this adaptive oscillator is however able to predict the state evolution (and the evolution of higher order derivatives) in real-time, i.e. without delay with respect to the measured output. In counterpart, this filtering oscillator is designed to work only for quasi-sinusoidal signals, and would not work properly with other profiles, although adaptations can be found in [17], [18].

The adaptive characteristics of this algorithm refers to its ability to continuously adapt to changes in the input signal features, namely the movement phase, frequency, amplitude, and offset, in the case of a sinusoidal input. It is a system of differential equations based on a Hopf oscillator that can synchronize on a periodic input $F(t)$ :

$$
\begin{aligned}
& \dot{x}(t)=\gamma\left(\mu-\left(x(t)^{2}+y(t)^{2}\right)\right) x(t)+\omega(t) y(t)+v F(t), \\
& \dot{y}(t)=\gamma\left(\mu-\left(x(t)^{2}+y(t)^{2}\right)\right) y(t)-\omega(t) x(t)
\end{aligned}
$$

where $x(t)$ and $y(t)$ are the oscillator's states; $\omega(t)$ is the oscillator intrinsic frequency; and $\mu$ and $\gamma$ determine the oscillator amplitude and the attractivity of the limit cycle, respectively. In the present paper, we used $\mu=1$ (such that the oscillator's intrinsic amplitude equals one) and $\gamma=8$ (like in [13], [18]). The learning parameter $v$ determines the speed of the phase synchronization with respect to $F(t)$. If this oscillator is augmented with a third state variable to learn the frequency of the input signal $F(t)$ (instead of doing only mere synchronization), Righetti et al. [13] proved that the basin of attraction becomes infinite, such that any periodic signal can be learned from any initial condition. This is done by using an integrator whose argument sums up to zero over one period if $F(t)$ and $y(t)$ have a phase-lag of $90^{\circ}$ (i.e. if $F(t)$ and $x(t)$ are in phase):

$$
\dot{\omega}(t)=v F(t) \frac{y(t)}{\sqrt{x(t)^{2}+y(t)^{2}}} .
$$

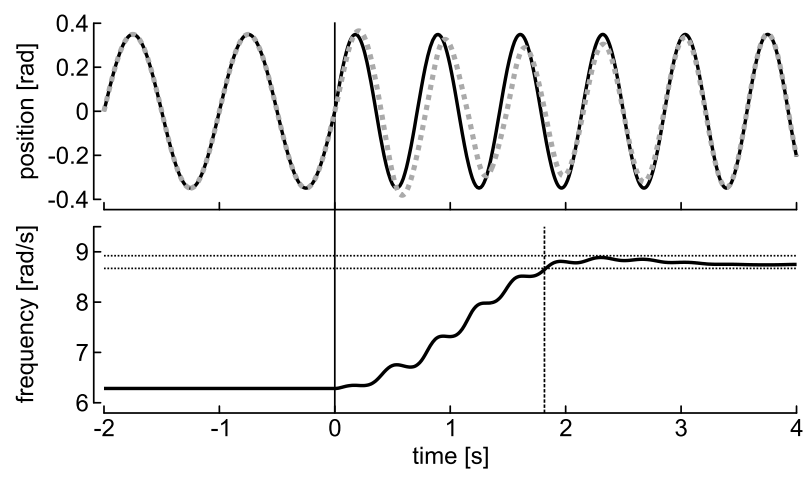

Fig. 2. Example of the oscillator's adaptation dynamics. Top panel: the oscillator's output $\hat{\theta}(t)$ (dotted gray line) filters out the sudden change in the input $\theta(t)$ (solid black line), i.e. a frequency step at $t=0$. Bottom panel: corresponding evolution of the learned frequency $\omega(t)$.

In the present paper, we moreover implemented a mechanism to reset the integrator (2) to $\omega=2 \pi$ if $\omega<0$, in order to avoid the tracking of negative or near-zero frequencies. Visual inspection of the data revealed that this happened very rarely.

Finally, [17] proposed to use this building block to learn the parameters of a (multi-)sinusoidal input by using the difference between the actual signal $\theta(t)$ and the filtered (or learned) signal $\hat{\theta}(t)$ as input, i.e. $F(t)=\theta(t)-\hat{\theta}(t)$. The filtered signal is simply the oscillator output plus an offset term, i.e.:

$$
\hat{\theta}(t)=\alpha_{0}(t)+\alpha_{1}(t) x(t),
$$

where the amplitude $\alpha_{1}$ and the offset $\alpha_{0}$ can be learned by integrators:

$$
\begin{aligned}
& \dot{\alpha}_{0}(t)=\eta F(t), \\
& \dot{\alpha}_{1}(t)=\eta x(t) F(t),
\end{aligned}
$$

with $\eta$ being the integrator gain.

Assuming that the actual input signal is (quasi-)sinusoidal, the state estimator block easily provides a zero-delay filtered estimate of the input signal (3), but also of its velocity and acceleration:

$$
\begin{aligned}
& \hat{\hat{\theta}}(t)=\alpha_{1}(t) \omega(t) y(t), \\
& \hat{\hat{\theta}}(t)=-\alpha_{1}(t) \omega(t)^{2} x(t) .
\end{aligned}
$$

As an example, Figure 2 shows the oscillator adaptation to a step change in the input frequency. It is visible that the oscillator acts like a classical low-pass filter, but on the signal features (the frequency in this case), rather than on the signal itself.

\section{HEALTHY HUMAN IN THE LOOP: SIMULATION AND EXPERIMENT}

Here we explore the feasibility of doing human assistance and rehabilitation using the adaptive oscillator presented above. In particular, this paper deals with the augmentation of a single degree-of-freedom, namely the human elbow. The fundamental building blocks of the coupled system (human elbow + exoskeleton) are depicted in Figure 3 and described below. 


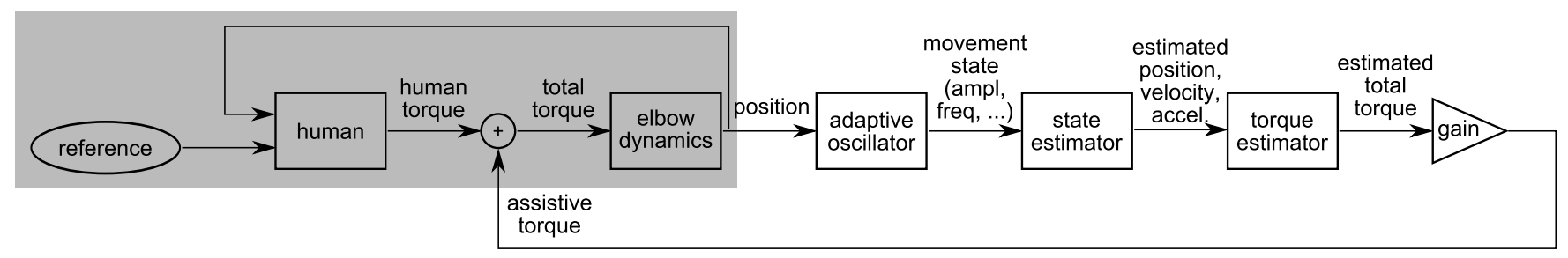

Fig. 3. Block diagram of the integrated system (human elbow + exoskeleton). The shaded part (associated with the human controller and elbow dynamics) needs to be modeled only for simulations, and not for the actual implementation of the assistance algorithm. Each box is detailed in the text.

\section{A. Elbow dynamics and torque estimator}

The elbow dynamics map the input torque to an output trajectory, therefore integrating the different forces acting at the joint level. We propose to capture these dynamical interactions with a simple model of a damped pendulum:

$$
I \ddot{\theta}(t)=-m g l \sin \theta(t)-b \dot{\theta}(t)+u(t),
$$

where $I, m$, and $l$ denote respectively the forearm+hand inertia, mass, and equivalent length; $b$ denotes the elbow viscous damping constant; $g=9.81 \mathrm{~m} / \mathrm{s}^{2}$ denotes the constant of gravity; and $\theta(t), \dot{\theta}(t)$, and $\ddot{\theta}(t)$ denote the elbow angular position, velocity, and acceleration, respectively. Finally, $u(t)$ denotes the input torque that is applied at the elbow joint, both by the user $u_{h}(t)$ and by the assistance device $u_{e}(t)$, i.e. $u(t)=u_{h}(t)+u_{e}(t)$. Note that the assistance device mass was assumed to be negligible with respect to the human forearm. As shown in Figure 3, this model is only used for simulations, and not for the actual experiments.

The torque estimator block simply retrieves an estimate of the total torque $\hat{u}(t)$ based on an inverse dynamical model of (6), i.e.:

$$
\hat{u}(t)=m g l \sin \hat{\theta}(t)+b \hat{\dot{\theta}}(t)+I \hat{\ddot{\theta}}(t)
$$

where $\hat{\theta}(t), \hat{\dot{\theta}}(t)$, and $\hat{\ddot{\theta}}(t)$ are provided by (3) and (5).

Finally, a fraction of this torque is fed back to the user via the assistance device, i.e.:

$$
u_{e}(t)=\kappa \hat{u}(t)
$$

where $0 \leq \kappa<1$ is the assistance gain. Assuming a stationary sinusoidal movement and a perfect inverse dynamical model (7), such that $\hat{u}(t)=u(t)$, the total torque should emerge from a collaboration between the user $(1-\kappa)$ and the assistance device $(\kappa)$. The fundamental building block being an adaptive oscillator (1), this assistance algorithm is characterized by a certain degree of inertia, which is tuned by setting the gains $v$ and $\eta$. However, proper tuning of these gains should allow the user to smoothly drive the oscillator adaptation, such that the user will keep the control of all "high-level" parameters (movement amplitude, frequency, and offset) while feeling the oscillator synchronized with his/her movements to make them easier to perform. For both the simulations and experiments reported in this paper, we used $v=20$ and $\eta=5$ as a compromise between good reactivity and low risk of instability.

\section{B. Human-in-the-loop: simulation}

Simulations of the behavior with a healthy participant were carried out in order to explore the algorithm properties for preparing the actual experiments. Altering the healthy human controller also allows to make hypotheses about the system's behavior with patients affected by specific disorders (see Section IV).

The human controller of Figure 3 receives two inputs: (i) a reference specifying the desired movement to perform; and (ii) the actual position (and possibly other movement-related variables) fed back from the sensory apparatus. As output, this block provides the torque $u_{h}(t)$ that is applied by the human at the joint level to match the actual movement with the desired one. A crude model of this block will be proposed for simulations, but this model is actually not required for our application, neither are specific assumptions about the way the reference is internally represented (as a whole trajectory, or only specific landmarks, or something in between), or which sensory variables are actually used, i.e. the shaded area in Figure 3.

The task of the simulated human is simply to oscillate the upper-arm about the elbow and around the upright vertical position (same posture as in the actual experiment, see Figure $5)$. We crudely assume that this reference trajectory is internally represented as a whole, i.e. $\theta_{\text {ref }}(t)=A_{\text {ref }} \sin \left(\omega_{\text {ref }} t\right)+$ $\pi$, and that the human stabilizes it using a simple PID controller:

$$
u_{h}(t)=k_{p} e(t)+k_{i} \int e(t) d t+k_{d} \dot{e}(t)
$$

where $k_{p}=100, k_{i}=5$, and $k_{d}=2$ are the controller gains, and $e(t)=\theta_{\text {ref }}(t)-\theta(t)$ is the error signal. We thus assume that the human is able to sense the elbow position without delay and noise. These assumptions about the control mechanisms are very likely far from the reality. For instance, it is postulated that the control of rhythmic movements with the upper limbs is also managed by low-level neural structures based on oscillators (CPG) [19], [20], [21], [22]. However, these differences are not crucial to illustrate how our method works, since the assistance algorithm retrieves the torque applied at the joint level, disregarding the neural structure and mechanisms that produced it.

The physiological parameters were set as follows: forearm equivalent mass $m=1.8 \mathrm{~kg}$, forearm equivalent length $l=$ $0.2 \mathrm{~m}$, forearm inertia $I=0.11 \mathrm{Nms}^{2} / \mathrm{rad}$, and elbow damping $b=0.4 \mathrm{Nms} / \mathrm{rad}$. 


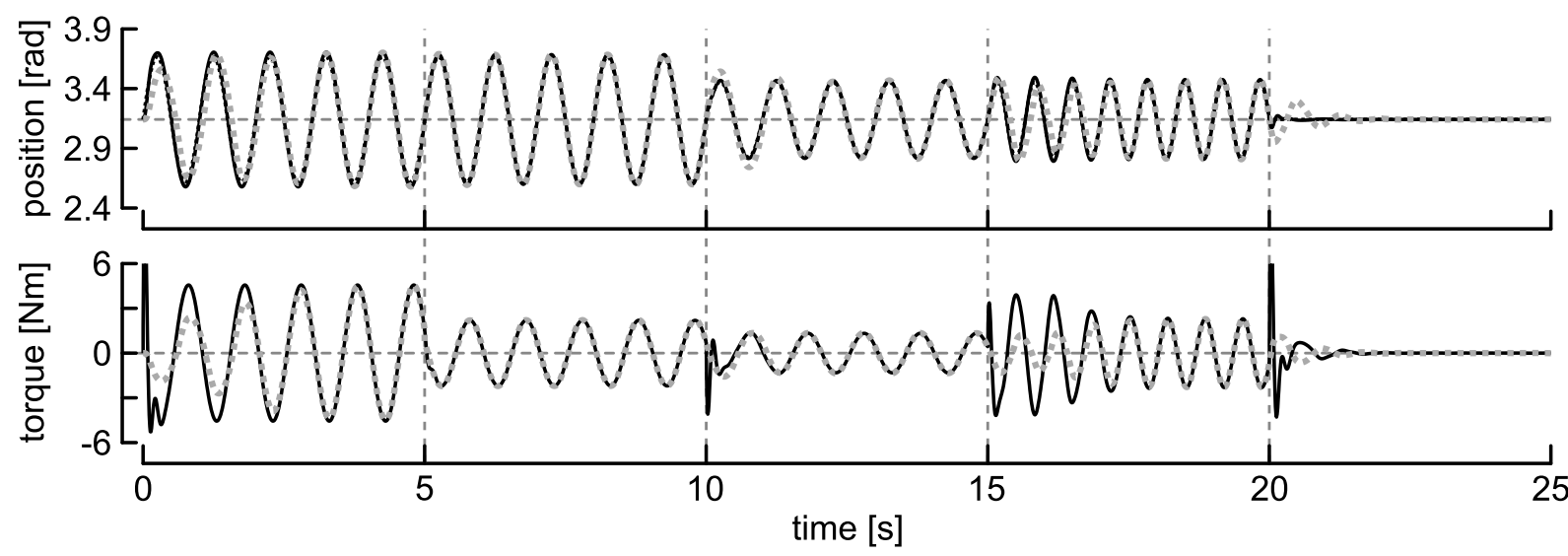

Fig. 4. Simulation of elbow assistance with an healthy participant. Top panel, elbow position: reference $\theta_{r e f}(t)$ (dotted black), actual $\theta(t)$ (solid black), and estimated $\hat{\theta}(t)$ (dotted gray). Bottom panel, applied torque: human $u_{h}(t)$ (solid black) and estimate of the human, i.e. (1 $\left.-\kappa\right) \hat{u}(t)$ (dotted gray). The assistance torque $u_{e}(t)$ is zero for $t<5 \mathrm{~s}$ and equal to $\kappa \hat{u}(t)=(1-\kappa) \hat{u}(t)$ (since $\kappa=0.5$ in this case) otherwise. The task is described in the text.

Simulation results are shown in Figure 4: the reference trajectory $\theta_{r e f}(t)$ is the dotted black line, and the actual trajectory is the solid black line. These are hardly distinguishable due to the good tracking capacity of the PID controller. More interestingly, the bottom panel shows the torque that was applied by the human to realize this tracking, i.e. $u_{h}(t)$ (solid black), and an estimate of this torque obtained from (7) (dotted gray). Except during transients, these two signals were also pretty overlapped, demonstrating the tracking capacity of the adaptive oscillator. The task was as follows:

- $0 \leq t<5$ : no assistance was applied $(\kappa=0)$ and the oscillator converged to the actual dynamics. $A_{r e f}=\pi / 6$, $\omega_{\text {ref }}=2 \pi$.

- $5 \leq t<10: 50 \%$ of assistance was applied $(\kappa=0.5$, this was the case until the end). $A_{r e f}=\pi / 6, \omega_{r e f}=2 \pi$. The human torque significantly decreased, showing the effect of assistance.

- $10 \leq t<15$ : switch in the reference amplitude: $A_{\text {ref }}=$ $\pi / 10, \omega_{\text {ref }}=2 \pi$. The oscillator reacted quickly, such that the transient was very smooth.

- $15 \leq t<20$ : switch in the reference frequency: $A_{\text {ref }}=$ $\pi / 10, \omega_{\text {ref }}=3 \pi$. The oscillator took a bit longer to adapt, but the transient was again smooth.

- $20 \leq t$ : the task instruction was to stop the movement $\left(A_{\text {ref }}=0\right)$, such that the assistance torque rapidly died out.

Overall, this simulation shows the capacity of our assistance method to reduce the human effort, without depriving him/her to fully control the movement features, like amplitude and frequency.

\section{Human-in-the-loop: experiment}

In this section, we report preliminary experimental results that were obtained with human participants.

1) Experimental setup and protocol: The assistance device we used in this experiment was the NEUROExos (Figure 5), an elbow active orthosis conceived for neurorehabilitation and assistive purposes [23]. NEUROEXOS relies on three main features: (i) a four degrees of freedom (DOF) passive

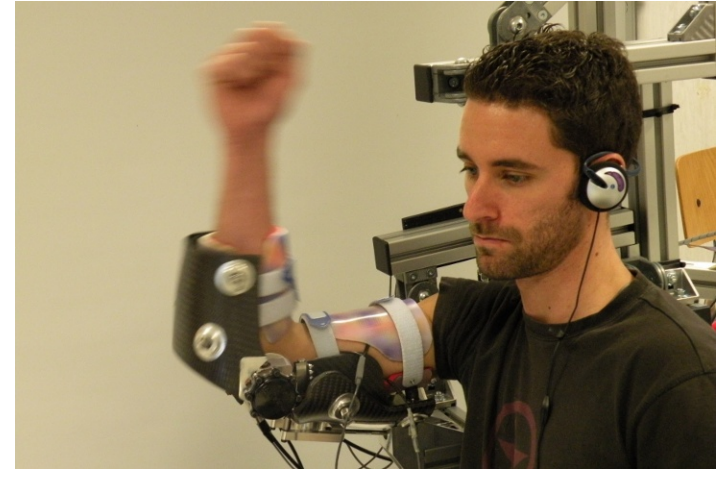

Fig. 5. Front view of a participant wearing the NEUROExos.

mechanism, conceived to automatically align the exoskeleton and elbow rotation axes, (ii) a double-shell structure of the links, providing a versatile, comfortable and distributed userrobot mechanical interface, and (iii) an antagonistic tendondriven compliant actuation, allowing a near-zero impedance torque control using force sensors [24], with a $-3 \mathrm{~dB}$ bandwidth of about $15 \mathrm{~Hz}$.

In order to monitor the participant's effort associated with movement performance during all conditions, we recorded the surface EMG activity from the biceps brachii and triceps brachii muscle using bipolar surface $\mathrm{Ag} / \mathrm{AgCl}$ electrodes (Pirronse\&Co., Italy) attached about $2 \mathrm{~cm}$ apart along the longitudinal axis of the muscle belly. All the EMG recordings were digitized at $1 \mathrm{kHz}$ using the Telemyo 2400R G2 Analog Output receiver (Noraxon USA Inc., AZ, USA). EMG analog recordings and NEUROExos outputs were synchronized by means of a Labview routine running at $1 \mathrm{kHz}$ on a real-time controller NI PXI-8196 (National Instrument, TX, USA). A Labview routine was also developed to provide the participant with visual feedback and acoustic cueing (see later).

The participants (right-handed) comfortably sat on a chair, and wore the NEUROExos on their right arm. NEUROExos was fastened both at the participant upper arm and forearm using the custom-made double shell structure. The NEU- 


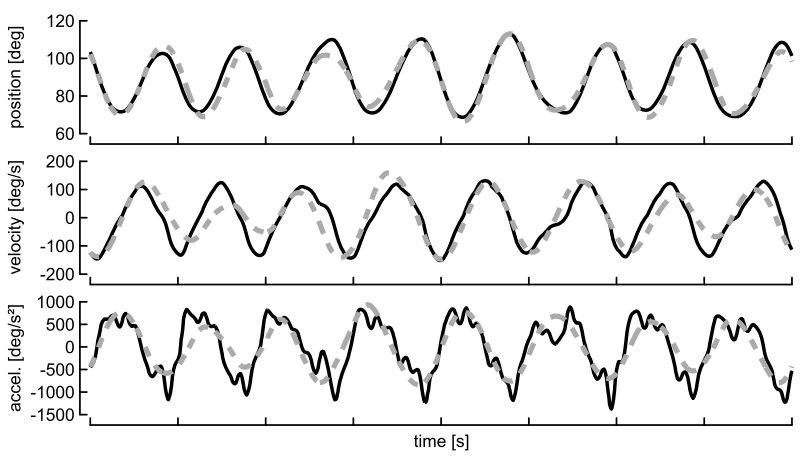

Fig. 6. Comparison between the actual kinematics (solid, black) and the filtered version provided by the adaptive oscillator (dashed, gray) during 8s of performance for a representative participant: position (top), velocity (middle) and acceleration (bottom).

ROExos support was adjusted to support the participant arm in the horizontal position, i.e. the shoulder forming an angle of about $90^{\circ}$ with respect to the chest in both frontal and transverse planes. Participants were asked to put their forearm in the upright vertical position - mimicking thus the inverse pendulum configuration of the limb during walking - and to make cyclical flexion/extension around this position at an amplitude and pace that was specified by the experimenter. The reference amplitude was kept constant across all conditions investigated here $\left(20^{\circ}\right.$, thus corresponding to a total elbow excursion of about $40^{\circ}$ ). This was softly constrained to the participant by giving him/her augmented visual feedback about the movement on a computer screen: a central cursor moved vertically by following the elbow angular displacement, while two peripheral cursors delimited the fixed movement range (corresponding thus to $\pm 20^{\circ}$ ). The movement pace was constant at $1 \mathrm{~Hz}$ and was softly constrained by a metronome ( 1 beep per cycle). Participants heard the metronome through headphones and were asked to synchronize with it. Both the visual feedback and the auditory cueing only provided guidance to the participant to follow the movement features. No corrective actions were applied by the exoskeleton to compensate for errors in movement amplitude or frequency, such that the participant always kept the full control of these high-level parameters.

2) Experimental results: The adaptive oscillator provided delay-free and filtered estimates of the elbow angular position, velocity and acceleration, according to (3) and (5). These estimates are compared to the actual profiles in Figure 6 , over $8 \mathrm{~s}$ of performance for a representative participant. This figure reveals the high performance of the filter based on an adaptive oscillator: the tracking is good, and the filtered signal is not lagging behind the actual one. Also, the oscillator output was a filtered version of the actual kinematics, which is particularly visible for the acceleration. Accordingly, we expect that the estimated torque is also a good estimate of the total torque, assuming that the inverse model (7) is accurate enough.

Looking into the details of possible kinematic changes across conditions, we computed the standard kinematic profiles over each cycle - limited by two successive velocity

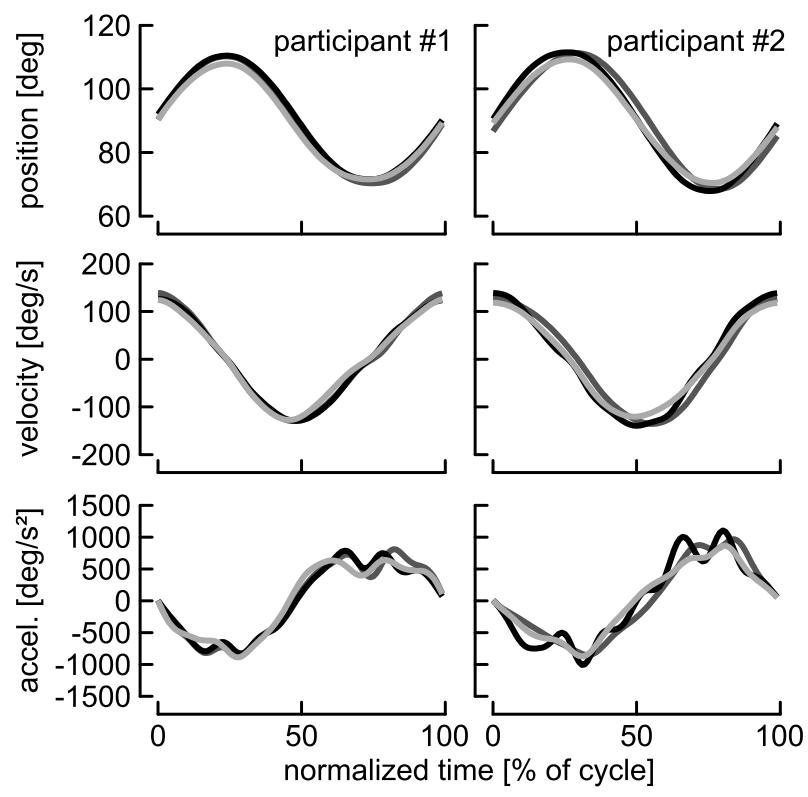

Fig. 7. For two participants, the panels show the steady-state kinematic profiles (position, top; velocity, middle; acceleration, bottom) without wearing the exoskeleton (light gray), and with the exoskeleton with two different assistance gains $(\kappa=0$, i.e. transparent mode, in dark gray; and $\kappa=0.5$, in black). These profiles were obtained by resampling the actual trajectories over 101 equally spaced points for each cycle, limited by two successive velocity peaks, then averaging for each of the 101 points across cycles.

peaks - by resampling over 101 equally spaced points (see Figure 7). This procedure was done for two different participants (Participant \#1 is the same as in Figure 6). We compared three conditions in steady-state, i.e. when the performance plateau was reached: (i) same movement/posture but without wearing the exoskeleton (the angular position of the elbow was measured using a goniometer); (ii) wearing the exoskeleton controlled in "transparent mode" (i.e. $\kappa=$ 0 ); and (iii) $50 \%$ of assistance provided (i.e. $\kappa=0.5$ ). The profiles look quite similar among conditions. The only marked difference being that the acceleration profile was more jerky when wearing the exoskeleton, this phenomenon being amplified when assistance was switched on. This is presumably due to high-frequency dynamics at the mechanical interface between the user and the exoskeleton.

The largest difference between the performance in these contexts was actually visible in the EMG profiles developed by the participants. Figure 8 shows the evolution of the mean normalized and rectified EMG for the biceps and triceps across the cycles of the same three different conditions and for the same two participants. Two important results are visible on this figure: (i) wearing the exoskeleton without assistance (difference between the condition without exoskeleton, light gray, and the transparent condition $\kappa=0$, dark gray) induced larger activity, mainly in the biceps, this being certainly due to the exoskeleton forearm's mass and inertia, that were not compensated in that mode and that mainly loaded the joint flexor; and (ii) providing assistance $(\kappa=0.5)$ induced a marked decrease in peak EMG, after about 10 to 20 cycles that were necessary to fully adapt to 

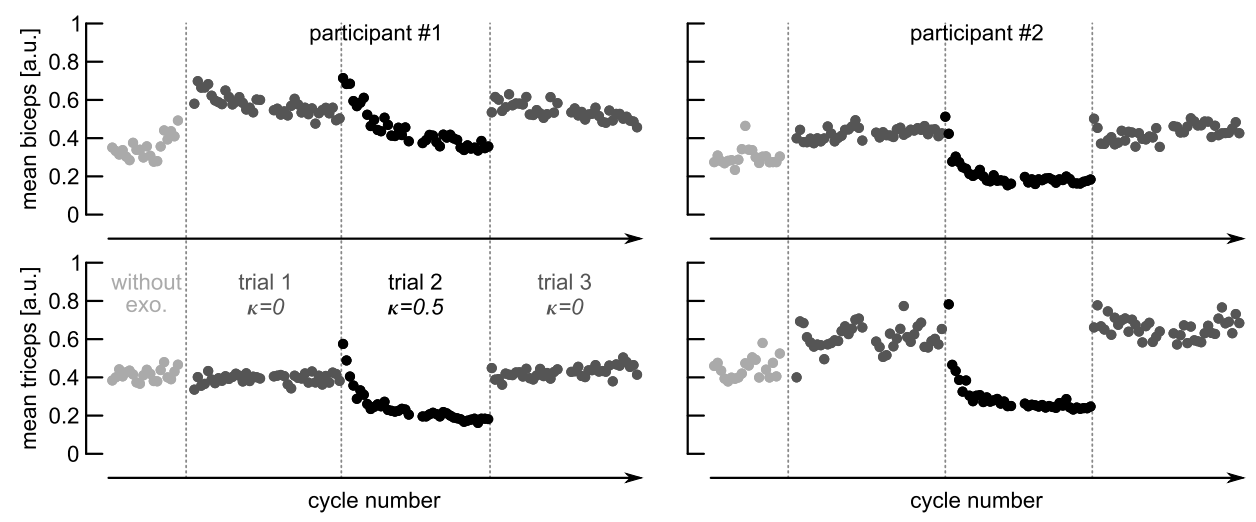

Fig. 8. For the same two participants, the panels show the average of the measured rectified EMG for the biceps (top panel) and triceps (bottom panel). The figure shows the last 20 cycles of the condition without wearing the exoskeleton (light gray), and the first and last 20 cycles of three trials wearing the exoskeleton (dark gray: trials 1 and 3 without assistance, i.e. $\kappa=0$; black: trial 2 with $50 \%$ of assistance provided, i.e. $\kappa=0.5$ ).

this assisted environment. The level of assistance we tested corresponded to a decrease in the mean EMG with respect to the condition without exoskeleton in three of the four EMG traces shown in Figure 8.

In sum, the assistance method proved to reduce the human effort through a marked decrease in biceps and triceps EMG, although the resulting influence on the movement kinematics was negligible. Further experiments are carried out to illustrate the method flexibility to the participant wishes, for instance by instructing him/her to modulate the movement amplitude and/or frequency on-line during execution. First results are promising and will be reported elsewhere.

\section{PATIENT WITH MUSCLE WEAKNESS: SIMULATION}

In this section, we aim at discussing the relevance of our method for designing novel rehabilitation programs for patients with movement disorders, e.g. due to muscle weakness or brain injury. The physiological parameters are the same as in Section III-B. The controller is also the same, except that we assume the simulated patient to be unable to produce elbow torque larger than $0.7 \mathrm{Nm}$ in magnitude ${ }^{2}$. For this reason, the task was simplified since the movement oscillated around the downward position (stable equilibrium). Since the focus was not on the capacity to adapt, only two conditions were tested: (i) $0 \leq t<5$ : no assistance was provided $(\kappa=0)$ and the oscillator converged to the actual dynamics. The reference was set with $A_{r e f}=\pi / 6, \omega_{r e f}=2 \pi$; and (ii) $5 \leq t$ : $50 \%$ of assistance was provided $(\kappa=0.5)$. Same reference signal.

Figure 9 shows the result of this simulation. Without assistance, the patient is unable to fulfill the task, due to torque saturations. As consequence, the stabilized movement is of much smaller amplitude. Once assistance is provided, the amplitude progressively reaches the reference one, such that the patient's applied torque eventually leaves the saturation regime after about $t=8 \mathrm{~s}$.

\footnotetext{
${ }^{2}$ This very low value was taken as a borderline to demonstrate the relevance of the proposed approach.
}

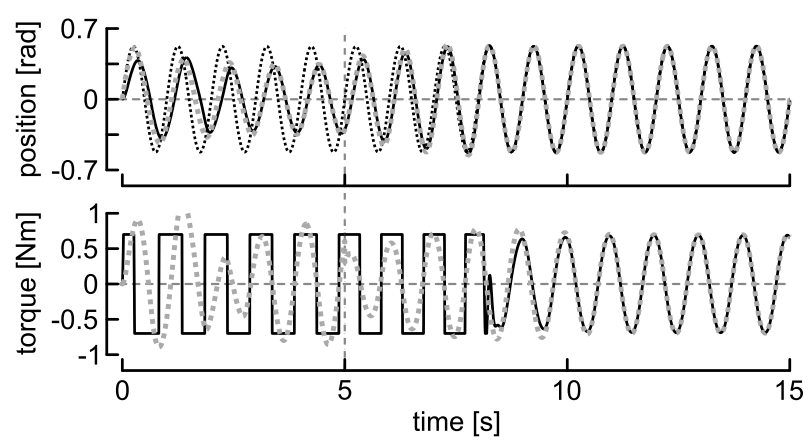

Fig. 9. Simulation of elbow assistance with a patient suffering from muscle weakness. Top panel, elbow position: reference $\theta_{\text {ref }}(t)$ (dotted black), actual $\theta(t)$ (solid black), and estimated $\hat{\theta}(t)$ (dotted gray). Bottom panel, applied torque: human $u_{h}(t)$ (solid black) and estimate of the human, i.e. $(1-\kappa) \hat{u}(t)$ (dotted gray). The assistance torque $u_{e}(t)$ is zero for $t<5 \mathrm{~s}$ and equal to $\kappa \hat{u}(t)=(1-\kappa) \hat{u}(t)$ (since $\kappa=0.5$ in this case) otherwise. The task is described in the text.

\section{CONCLUSION AND FUTURE WORK}

In the present paper, we proposed a new method for human assistance that exploits the intrinsic flexibility of adaptive oscillators. The method proved to be efficient for assisting rhythmic forearm movements about the elbow in healthy participants, both in simulations and - more importantly - in preliminary experiments with two participants. The results illustrated that not only the oscillator adapted to the participant behavior, but also that the participant adapted to the assisted regime, by reducing both biceps and triceps EMGs. Moreover, this adaptation was surprisingly fast, about 10 cycles, and required simple sensors (joint position) and crude modeling assumptions about the human controller (which was actually modeled for simulations only) and the joint dynamics (which were captured by a very simple equation). We are currently finalizing another paper to detail these experiments and to establish the statistical significance of our approach. This paper will also establish the task flexibility, by showing that participants keep the full control of the high-level movement features (amplitude, frequency).

Preliminary simulation results also illustrated that the method might be efficient for patients, mainly those suffering 
from muscle weakness. However, we still have to explore the validity of this claim with actual patients.

So far, the method focused on assistance of the forearm, although very few rhythmic movements are performed by the upper limbs in daily life, a notable exception being (simplified) juggling [25]. Therefore, the next step will be to prove the relevance of our approach for a more functional task, walking being the clear target. We will have to adapt the method presented here in order to (i) be adaptive to nonsinusoidal rhythmic movements; (ii) cope with the dynamics due to contacts with the ground; (iii) coordinate the multiple degrees of freedom involved in locomotion; and (iv) explore alternatives to the need of deriving an inverse dynamical model, that is more tedious to get for locomotion tasks.

\section{REFERENCES}

[1] S. Hesse, H. Schmidt, C. Werner, and A. Bardeleben, "Upper and lower extremity robotic devices for rehabilitation and for studying motor control." Curr Opin Neurol, vol. 16, no. 6, pp. 705-710, Dec 2003. [Online]. Available: http://dx.doi.org/10.1097/01.wco.0000102630.16692.38

[2] A. Dollar and H. Herr, "Lower extremity exoskeletons and active orthoses: Challenges and state-of-the-art," Robotics, IEEE Transactions on, vol. 24, no. 1, pp. 144-158, Feb. 2008.

[3] D. P. Ferris, "The exoskeletons are here." J Neuroeng Rehabil, vol. 6, p. 17, 2009. [Online]. Available: http://dx.doi.org/10.1186/1743-00036-17

[4] E. T. Wolbrecht, V. Chan, D. J. Reinkensmeyer, and J. E. Bobrow, "Optimizing compliant, model-based robotic assistance to promote neurorehabilitation." IEEE Trans Neural Syst Rehabil Eng, vol. 16, no. 3, pp. 286-297, Jun 2008. [Online]. Available: http://dx.doi.org/10.1109/TNSRE.2008.918389

[5] H. Vallery, E. H. F. van Asseldonk, M. Buss, and H. van der Kooij, "Reference trajectory generation for rehabilitation robots: complementary limb motion estimation." IEEE Trans Neural Syst Rehabil Eng, vol. 17, no. 1, pp. 23-30, Feb 2009. [Online]. Available: http://dx.doi.org/10.1109/TNSRE.2008.2008278

[6] G. Colombo, M. Wirz, and V. Dietz, "Driven gait orthosis for improvement of locomotor training in paraplegic patients." Spinal Cord, vol. 39, no. 5, pp. 252-255, May 2001. [Online]. Available: http://dx.doi.org/10.1038/sj.sc.3101154

[7] K. P. Westlake and C. Patten, "Pilot study of lokomat versus manual-assisted treadmill training for locomotor recovery poststroke." J Neuroeng Rehabil, vol. 6, p. 18, 2009. [Online]. Available: http://dx.doi.org/10.1186/1743-0003-6-18

[8] R. A. Scheidt, J. B. Dingwell, and F. A. Mussa-Ivaldi, "Learning to move amid uncertainty," J Neurophysiol, vol. 86, no. 2, pp. 971-985, Aug 2001.

[9] R. Riener, L. Lünenburger, S. Jezernik, M. Anderschitz, G. Colombo, and V. Dietz, "Patient-cooperative strategies for robot-aided treadmill training: first experimental results." IEEE Trans Neural Syst Rehabil Eng, vol. 13, no. 3, pp. 380-394, Sep 2005. [Online]. Available: http://dx.doi.org/10.1109/TNSRE.2005.848628

[10] E. H. F. Van Asseldonk, R. Ekkelenkamp, J. F. Veneman, F. C. T. Van der Helm, and H. van der Kooij, "Selective control of a subtask of walking in a robotic gait trainer(lopes)," in Proc. IEEE 10th International Conference on Rehabilitation Robotics ICORR 2007, Jun. 13-15, 2007, pp. 841-848.

[11] Y. Sankai, "Leading edge of cybernics: Robot suit hal," in Proc. International Joint Conference SICE-ICASE, Oct. 2006, pp. P-1-P-2.

[12] E. Guizzo and H. Goldstein, "The rise of the body bots," IEEE Spectrum, vol. 42, no. 10, pp. 50-56, Oct. 2005.

[13] L. Righetti, J. Buchli, and A. J. Ijspeert, "Dynamic hebbian learning in adaptive frequency oscillators," Physica D, vol. 216, pp. 269-281, 2006.

[14] J. Buchli, L. Righetti, and A. J. Ijspeert, "Frequency analysis with coupled nonlinear oscillators," Physica D, vol. 237, pp. 1705-1718, 2008.

[15] L. Righetti, J. Buchli, and A. J. Ijspeert, "Adaptive frequency oscillators and applications," The Open Cybernetics and Systemics Journal, vol. 3, pp. 64-69, 2009.
[16] A. Frigon and S. Rossignol, "Experiments and models of sensorimotor interactions during locomotion," Biol Cybern, vol. 95, no. 6, pp. 607627, 2006.

[17] L. Righetti and A. J. Ijspeert, "Programmable central pattern generators: an application to biped locomotion control," in Proc. IEEE International Conference on Robotics and Automation ICRA 2006, May 15-19, 2006, pp. 1585-1590.

[18] A. Gams, A. J. Ijspeert, S. Schaal, and J. Lenarčič, "On-line learning and modulation of periodic movements with nonlinear dynamical systems," Auton Robot, vol. 27, pp. 3-23, 2009.

[19] S. Schaal, D. Sternad, R. Osu, and M. Kawato, "Rhythmic arm movement is not discrete," Nat Neurosci, vol. 7, no. 10, pp. 1136$1143,2004$.

[20] E. P. Zehr and J. Duysens, "Regulation of arm and leg movement during human locomotion." Neuroscientist, vol. 10, no. 4, pp. 347-361, Aug 2004. [Online]. Available: http://dx.doi.org/10.1177/1073858404264680

[21] O. White, Y. Bleyenheuft, R. Ronsse, A. M. Smith, J.-L. Thonnard, and P. Lefèvre, "Altered gravity highlights central pattern generator mechanisms." J Neurophysiol, vol. 100, no. 5, pp. 2819-2824, Nov 2008. [Online]. Available: http://dx.doi.org/10.1152/jn.90436.2008

[22] R. Ronsse, D. Sternad, and P. Lefèvre, "A computational model for rhythmic and discrete movements in uni- and bimanual coordination." Neural Comput, vol. 21, no. 5, pp. 1335-1370, May 2009. [Online]. Available: http://dx.doi.org/10.1162/neco.2008.03-08-720

[23] T. Lenzi, S. De Rossi, N. Vitiello, A. Chiri, S. Roccella, F. Giovacchini, F. Vecchi, and M. C. Carrozza, "The neuro-robotics paradigm: NEURARM, NEUROExos, HANDEXOS," in Proc. Annual International Conference of the IEEE Engineering in Medicine and Biology Society EMBC 2009, Sep. 3-6, 2009, pp. 2430-2433.

[24] G. A. Pratt and M. M. Williamson, "Series elastic actuators," in Proc. IEEE/RSJ International Conference on Intelligent Robots and Systems 95. 'Human Robot Interaction and Cooperative Robots', vol. 1, Aug. 5-9, 1995, pp. 399-406.

[25] R. Ronsse, K. Wei, and D. Sternad, "Optimal control of a hybrid rhythmic-discrete task: the bouncing ball revisited." J Neurophysiol, vol. 103, no. 5, pp. 2482-2493, May 2010. [Online]. Available: http://dx.doi.org/10.1152/jn.00600.2009 